\title{
Article \\ A Computational Model for Pain Processing in the Dorsal Horn Following Axonal Damage to Receptor Fibers
}

\author{
Jennifer Crodelle ${ }^{1, *,+}$ and Pedro D. Maia ${ }^{2,+} \mathbb{D}$ \\ 1 Department of Mathematics, Middlebury College, Middlebury, VT 05753, USA \\ 2 Department of Mathematics, University of Texas at Arlington, Arlington, TX 76019, USA; \\ pedro.maia@uta.edu \\ * Correspondence: jcrodelle@middlebury.edu \\ + Both authors contributed equally to this work.
}

Citation: Crodelle, J.; Maia, P.D. A Computational Model for Pain Processing in the Dorsal Horn Following Axonal Damage to Receptor Fibers. Brain Sci. 2021, 11, 505. https://doi.org/10.3390/ brainsci11040505

Academic Editors: Michela Chiappalone and Marianna Semprini

Received: 19 March 2021

Accepted: 13 April 2021

Published: 16 April 2021

Publisher's Note: MDPI stays neutral with regard to jurisdictional claims in published maps and institutional affiliations.

Copyright: (C) 2021 by the authors. Licensee MDPI, Basel, Switzerland. This article is an open access article distributed under the terms and conditions of the Creative Commons Attribution (CC BY) license (https:/ / creativecommons.org/licenses/by/ $4.0 /)$.

\begin{abstract}
Computational modeling of the neural activity in the human spinal cord may help elucidate the underlying mechanisms involved in the complex processing of painful stimuli. In this study, we use a biologically-plausible model of the dorsal horn circuitry as a platform to simulate pain processing under healthy and pathological conditions. Specifically, we distort signals in the receptor fibers akin to what is observed in axonal damage and monitor the corresponding changes in five quantitative markers associated with the pain response. Axonal damage may lead to spike-train delays, evoked potentials, an increase in the refractoriness of the system, and intermittent blockage of spikes. We demonstrate how such effects applied to mechanoreceptor and nociceptor fibers in the pain processing circuit can give rise to dramatically distinct responses at the network/population level. The computational modeling of damaged neuronal assemblies may help unravel the myriad of responses observed in painful neuropathies and improve diagnostics and treatment protocols.
\end{abstract}

Keywords: pain processing; dorsal horn; neuronal dynamics; computational model; axonal damage; painful neuropathies

\section{Introduction}

Pain is the most common patient complaint in medical consultations and an experience that we will all have at some point during our life [1]. Our pain-processing mechanism emerges from a complex interplay of cognitive and affective processes with neurochemical and neuroanatomic systems [2]. The dorsal horn (DH) is an area of the spinal cord that plays a central role in processing nociceptive, or painful, signals with the midbrain and cortex providing top-down modulation to that circuitry [3]. As a consequence, this region is a common target for analgesic action and is thought to undergo changes that contribute to the exaggerated pain felt after nerve injury and inflammation [4]. For all its significance, the heterogeneity of various neuronal components of the dorsal horn circuitry continues to challenge our understanding of how we process painful sensory information.

Over the years, computational models of the neural activity associated with the DH gave rise to key insights regarding nociception-processing [5-8]. Most models posit that nociceptive activity is inhibited by $\mathrm{A} \beta$-fibers unless the activity in the C-fibers (painful stimuli) outweighs it, thus activating the peripheral nerves and create the experience of pain. While this gate control theory greatly simplifies the physiological mechanisms of pain processing [9-11], it does provide a reasonable starting point for most computational and modeling efforts. In this study, we use the model introduced by Crodelle et al. [12,13] as a platform to numerically simulate the population activity of projection, inhibitory, and excitatory neurons in the DH under healthy and pathological conditions.

The dynamics of injured neuronal assemblies is a topic of broad and current interest in computational neurology, with axonal damage and their distortions to the neural activity being a hallmark feature of traumatic brain injuries and degeneration [14-17]. While there 
are multiple mechanisms associated with axonal damage (such as focal axonal swellings or demyelination), they invariably alter the usual transmission of spike trains along neuronal fibers. Maia et al. [14] assembled a list of phenomenological input/output rules describing commonly-observed forms of spike train distortions, which led to several studies simulating the addition of an injured-neuron population to functional networks [18-23].

Having a computational model capable of simulating a broad repertoire of outcomes from injured neurons in the DH may provide new insight into abnormal pain processing, a symptom present in fibromyalgia, peripheral neuropathic pain, and other painrelated disorders [24,25]. In what follows, we model the effects of axonal damage [14] to mechanoreceptor and nociceptor fibers in the pain processing circuit [12,13] and illustrate how different types of axonal dysfunction give rise to distinct responses at the network/population level.

\section{Materials and Methods}

\subsection{Overview of Spinal Cord Model for Pain Processing}

In what follows, we utilize the firing-rate model for the processing of painful stimuli in the human spinal cord developed in [13]. The model is based on the widely-used gate-control theory of pain [9]. Shortly, projection neurons ( $P$ in Figure $1 \mathrm{~A}$ ) receive input from excitatory $(E)$ and inhibitory $(I)$ interneurons, as well as directly from two types of afferent fibers: $A \beta$ fibers and $C$ fibers. While there are many different types of afferent fibers carrying sensory information about touch, pressure, itch, burning, and pain, in this model, we focus on the nociceptive pain processing circuit and refer to all fibers carrying mechanical touch information as $A \beta$ fibers and those carrying slow pain as $C$ fibers, as has been done previously $[5,13]$. Each population $(P, E$, and $I)$ responds to a weighted input of firing rates from input (presynaptic) populations as determined by individual nonlinear response curves in the formalism of $[26,27]$. The model also includes N-methyl-D-aspartate (NMDA) type synapses from the $C$ fibers to the $P$ neurons modeled as a modulation of the synaptic weight as a function of $P$-neuron firing rate. All model details can be found in [13] and Appendix A.

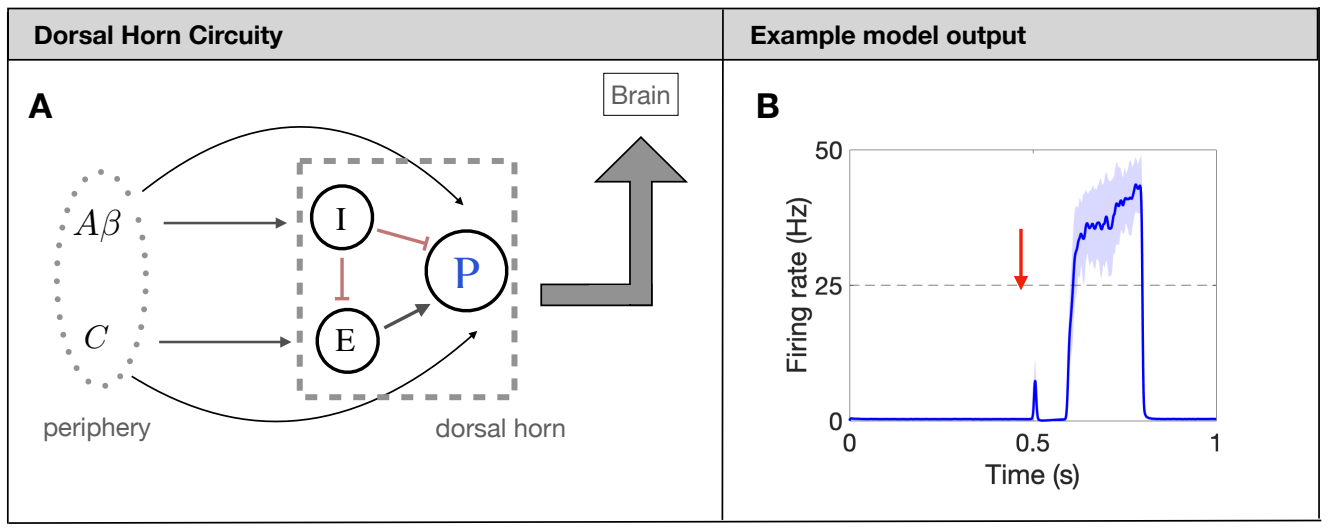

Figure 1. Firing-rate neural network model for pain processing. (A): Schematic of the spinal-cord circuitry. Projection neurons $[P$ in $(\mathbf{A})]$ receive input from excitatory $(E)$ and inhibitory $(I)$ interneurons, as well as directly from two types of afferent fibers: $A \beta$ fibers and $C$ fibers. (B): Typical output response (firing rate of $P$ neurons in (A)) of a healthy network to a brief painful stimuli at $0.5 \mathrm{~s}$.

The underlying mechanism for the model to produce a prototypical pain response, see Figure $1 \mathrm{~B}$, is the timing of the input from the $A \beta$ and $C$ fibers. Due to factors like axon myelination and diameter, the afferent fibers have significantly different conduction speeds leading to a distribution of arrival times in the DH of about 0-20 ms for $A \beta$ and 90-300 ms for $C$ [28]. To capture this characteristic, we introduce the delay and distribution on the Poisson spike trains for each fiber so that the DH output matches the experiments (again, see Figure 1B). Throughout the simulation, each fiber also has a spontaneous background 
firing rate of $1 \mathrm{~Hz}$ [29]. Finally, we average the Poisson spikes generated on each fiber (380 A $\beta$ fibers and $820 C$ fibers for a total of about 1200 as observed in one nerve bundle of rat $[30,31]$ ) over all fibers to yield an average firing rate for $A \beta$ fibers and $C$ fibers. This average firing rate for the fibers then serves as a weighted input to the nonlinear response functions of the $\mathrm{DH}$ populations. We measure the painful output of the model by quantifying characteristics of the response of the $P$ neurons during the time at which the $C$ fibers reach the DH (about $700 \mathrm{~ms}-750 \mathrm{~ms}$ ), which will be further explained in Section 2.4.

\subsection{Modeling Effects of Neuronal Injury to Spike-Train Activity}

Maia et al. [14] posited eight types of phenomenological input/output rules describing spike train distortions caused by the major forms of neuronal injury, including axonal swellings [15-17] and demyelination [32]. In their formulation, a spike train $\left\{x_{n}\right\}$ is transformed into another spike train $\left\{y_{n}\right\}$ according to some rule. If faithful conduction occurs, the input/output spike trains will match $\left(y_{n}=1 \cdot x_{n}\right)$. In the case of severe injury, the axonal impairment will delete all spikes in the train $\left(y_{n}=0 \cdot x_{n}\right)$. While these two cases would lead to trivial outcomes at the population level, some other types of distortion would not (see Figure 2B):

(i) Evoking potentials: In this rule, a single input spike triggers the formation of $k$ additional spikes.

(ii) Intermittent blocking: In this rule, the spike train switches between (total) blocking and normal conduction periodically (with period $f=2 \pi / \omega$ ).

(iii) Increasing refractoriness: In this rule, consecutive spikes may be deleted if the interspike interval between them is below $\tau$. This effectively increases the refractory period of the spike train.

We note that the latter impairment is strongly frequency-dependent; spike trains with higher firing rates are more strongly affected than spike trains with lower firing rates. This will ultimately lead to confusions of higher firing rates by lower ones. For more details regarding the mathematical formulation and numerical implementation of these rules, see [14].

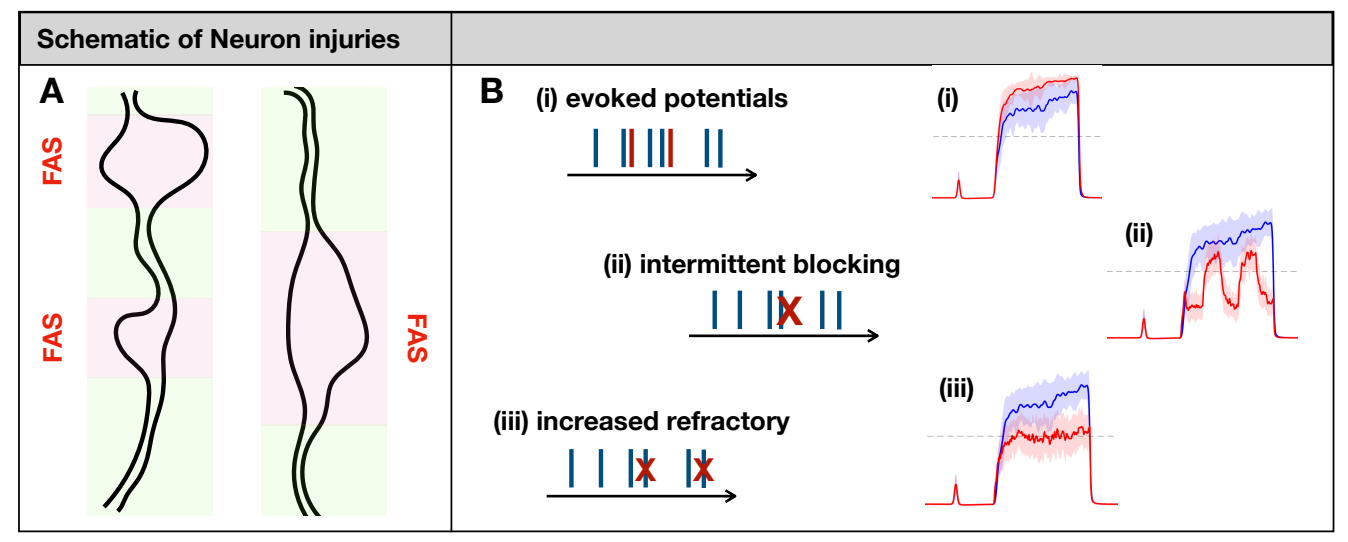

Figure 2. (A): Focal Axonal Swellings (FAS)—also called varicosities or beadings_-are enlarged, heterogeneous structures along axonal shafts that may dramatically distort conduction and synaptic transmission (see [16,33,34] and references therein). In our schematics, normal axonal segments are indicated in green, while the FAS portions are highlighted in pink/red. FAS are the most common forms of neuronal injury that may follow mechanical trauma or neurodegenerative disorders. (B): Previous computational modeling work characterized different ways in which FAS may affect spike train propagation [14-17]. In this work, we will simulate axonal injury by incorporating FAS effects that lead to (i) creation and (ii-iii) deletion of spikes. At the population level, neuronal injuries may lead to dramatically different pain-processing responses (in red) compared to the healthy system (in blue). 


\subsection{Injury Protocols for Receptor Fibers}

In this study, we opted to damage the receptor fibers ( $C$ and $A \beta$ fibers) with the injured rules listed above. The goal of our injury protocols is twofold: (i) to illustrate a large variety of potential outcomes at the collective level, and (ii) to study the effects of each parameter of the system. For the $C$-fibers, we introduce injured neurons to its population that distorts spike trains by evoking potentials, blocking spikes intermittently, and by increasing their refractoriness. For the $\mathrm{A} \beta$ fibers we introduce injured neurons that delay their spike trains by $d^{\star}$ ms. In our first simulations, we target the fiber populations separately, but in the last one, we consider a "mixed-effect" protocol where damaged $C$-fibers evoke potentials (that increases pain) concomitantly with spike train delays in the $\mathrm{A} \beta$ fibers (that reduces pain). This creates a "tug-of-war" between the injuries, and a state in which minor fluctuations could lead to significant oscillations in pain perception.

\subsection{Quantitative Markers for Pain Response}

It is challenging to quantify pain in a clinical setting since it involves cognitive and affective processes along with the patients' subjective assessments. To avoid ambiguities in this work, we characterize the pain response with the set of quantitative markers illustrated in Figure 3. The output of the model is the response that the projection neurons integrate from the DH and transmit to the cortex within the time interval $\left[t_{0}, t_{f}\right]$. The model was calibrated such that $\pi_{\text {thresh }}=25 \mathrm{~Hz}$ represents the firing-rate threshold for painful responses [12,13]. Figure 3 shows a typical painful response and depicts the following quantitative markers:

$$
\begin{aligned}
A_{\text {Total }} & =\text { Total area under the curve } \\
A^{*} & =\text { Area above } \pi_{\text {thresh }} \\
\pi^{*} & =\text { Average firing rate response, } \pi^{*}=A^{*} /\left|t_{f}-t_{0}\right| \\
\pi_{\max } & =\text { Maximum achieved firing rate } \\
N_{C} & =\text { number of times the painful response crosses } \pi_{\text {thresh }}
\end{aligned}
$$

The addition of injured fibers to the neuronal population may significantly alter the overall pain response and consequently, these four quantitative pain markers.

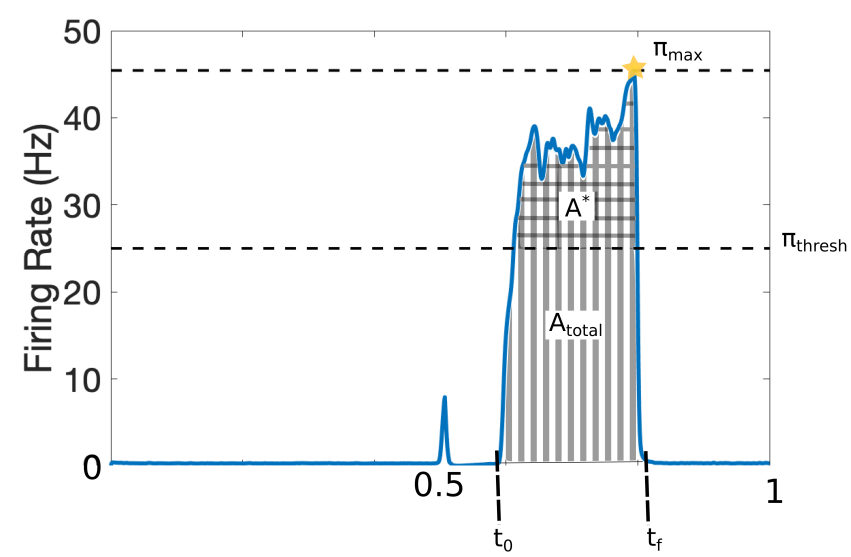

Time (s)

Figure 3. Quantitative markers for pain response: $\pi_{\text {thresh }}=25 \mathrm{~Hz}$ represents the firing-rate threshold for painful responses; $A^{*}$ (checked area) is the total area above that threshold, $A_{\text {Total }}$ (striped area) denotes the total area of the pain response (including above $\pi_{\text {thresh }}$ ); $t_{0}$ defines the initial time of pain response and $t_{f}$ the final time of the pain response; and $\pi_{\max }$ denotes the maximum achieved firing rate of the pain response. We also define the average firing-rate response, $\pi^{*}=A^{*} /\left|t_{f}-t_{0}\right|$ and the number of times $N_{C}$ that the signal crosses the pain threshold $\left(N_{C}=2\right.$, not depicted). 


\section{Results}

\subsection{Effects of Different Injuries on C Fibers}

Figure 4 illustrates how the painful response changes when populations of injured $\mathrm{C}$ fibers are introduced into the system. In these simulations, the injured neurons are all of the same type, i.e., we consider only one input/output rule to govern spike train distortion at a single time. The top panels in Figure 4 show the effect of the intermittent blocking injury with frequency of $\pi / 20$ (A) and $\pi / 40$ (B), respectively. In both cases, the switches between (total) blocking and normal conduction within the spike trains lead to oscillations in the overall response. The maximum achieved firing rate $\left(\pi_{\max }\right)$, the area of the curve above $25 \mathrm{~Hz}\left(A^{*}\right)$ and the average firing rate response $\left(\pi^{*}\right)$ all decay after injury. The injured responses also appear to be more irregular than the stereotypical response. The mid panels in Figure 4 show a contrasting case, where the pain response increases due to the evoked potentials (which may occur with a probability of $20 \%$ and $40 \%$ in Panels $\mathrm{C}$ and $\mathrm{D}$, respectively). In this case, $\pi_{\max }, A^{*}$ and $\pi^{*}$ increase proportionally to the evoked probability. Finally, the bottom panels in Figure 4 show the effects of the increased refractoriness injury type. In this rule, a consecutive spike is deleted if it's within $15 \mathrm{~ms}$ (Panel E) or $25 \mathrm{~ms}$ (Panel F) from the preceding spike, and as a consequence, all quantitative pain markers also decay.
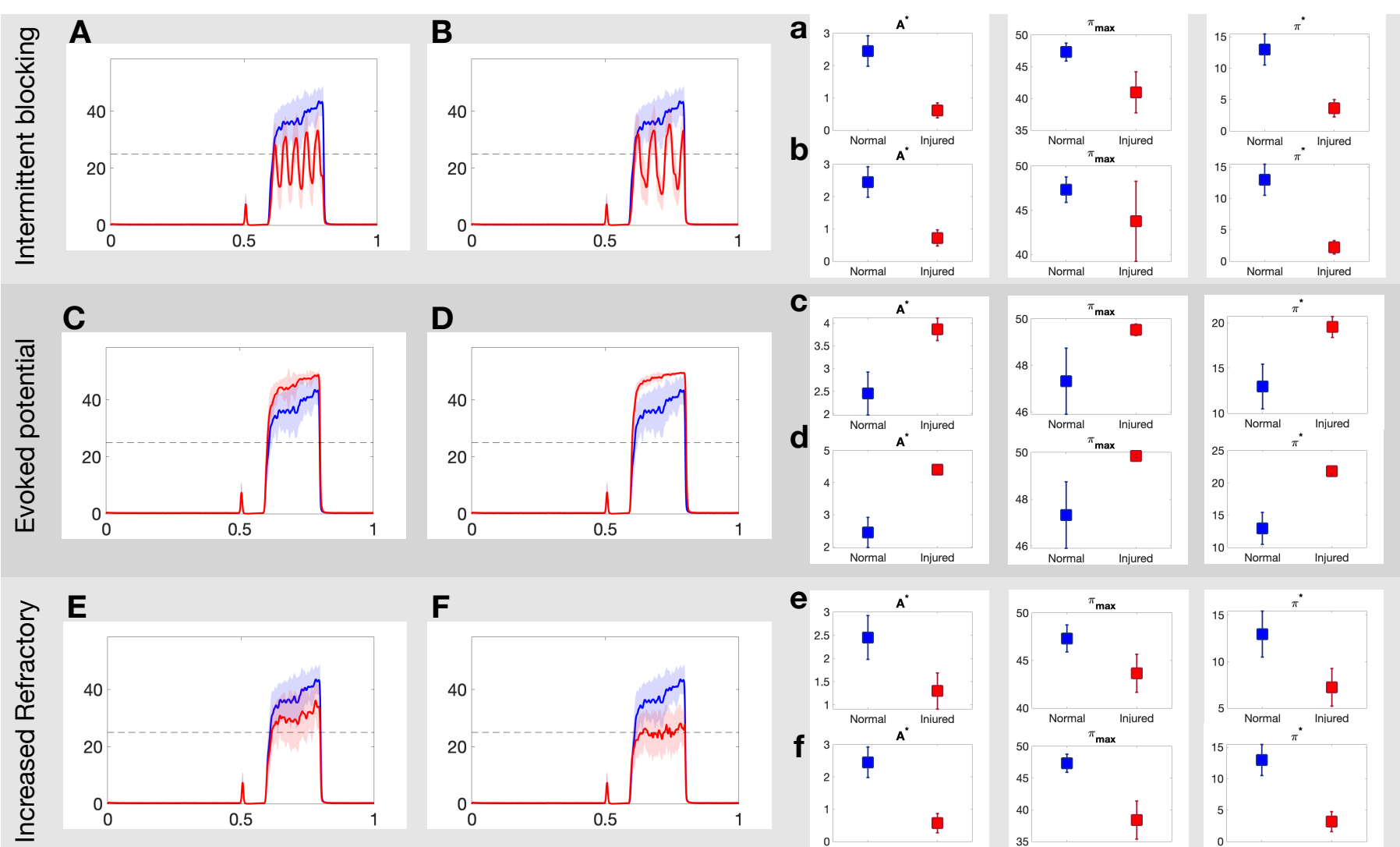

\section{$\mathbf{F}$}
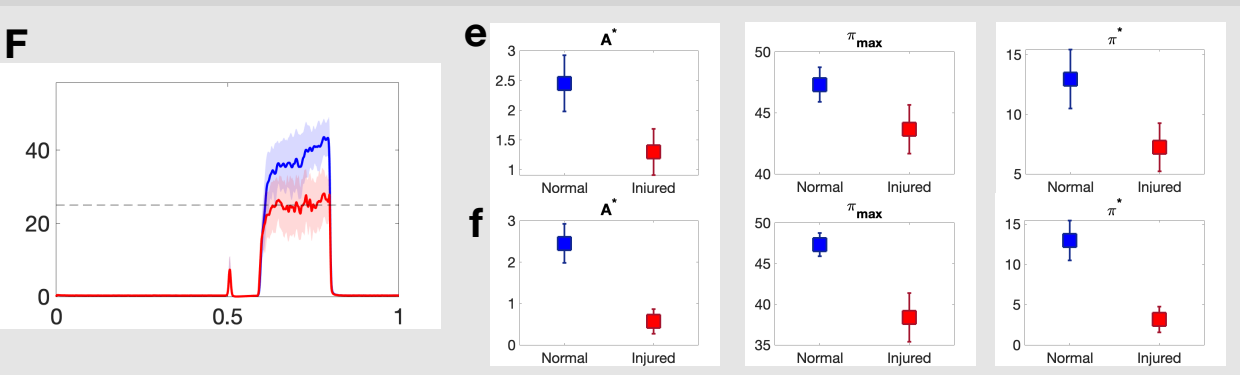

Figure 4. Example realizations of the DH projection neuron output to each of the three injuries on the C-fibers. (A,B): Projection neuron firing rate in response to intermittent blocking injury with frequency of $\pi / 20$ and $\pi / 40$, respectively; (C,D): evoked potential injury for an evoked probability of $20 \%$ and $40 \%$, respectively; and (E,F): increased refractory injury with a period of $15 \mathrm{~ms}$ and $25 \mathrm{~ms}$, respectively. See Section 2.2 for more details about each injury. (a-f): Three quantitative measures area above threshold, $A^{*}$; average firing rate response, $\pi^{*}$; number of threshold crossings of firing rate response, $N_{C}$, for each injury for normal (blue) and injured (red) responses in the projection neurons. See Section 2.4 for more details on each quantitative pain marker. 
Figure 5 expands on the qualitative results above by quantifying the markers for pain response as a function of the percentage of injured neurons in the targeted fiber population. The top panel of Figure 5 shows the result for intermittent blockage (with period $f=2 \pi / 10)$; we observe a monotonic decay in the parameters $\left[\pi^{*}, A^{*}, A^{0}, \pi_{\max }\right]$, but a maximum $N_{C}$ value is achieved near $25 \%$ injury level. The middle panel of Figure 5 shows analogous plots for the increased refractoriness injury (with $\tau=10 \mathrm{~ms}$ ). The markers follow a similar trend with the exception of $N_{C}$ that seems to exhibit a sigmoid-like shape. These two forms of injury contrast dramatically to the evoked potential injury shown in the bottom panel of Figure 5 . There, the parameters $\left[\pi^{*}, A^{*}, A^{0}, \pi_{\max }\right]$ increase monotonically while $N_{C}$ decays. Overall, these results show that different types of axonal injuries in the $C$-fibers can lead to dramatic differences in all quantitative markers of pain.

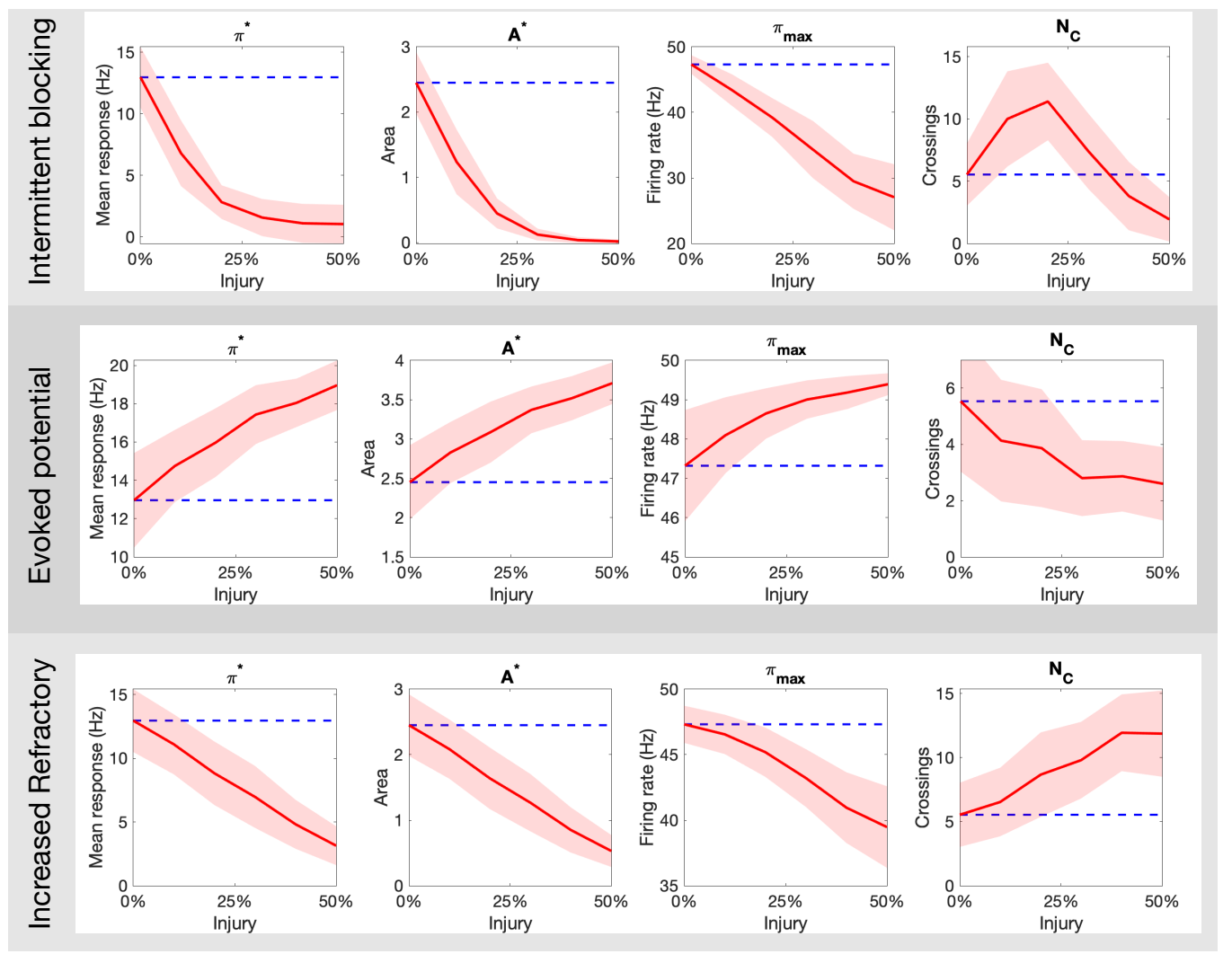

Figure 5. How the different pain quantitative markers $\left(\pi^{*}, A^{*}, A^{0}, \pi_{\max }\right.$ and $\left.N_{C}\right)$ change as a function of the type (intermittent blocking, evoked potentials, and increased refractory) and amount (\% of $C$ fibers damaged) of neuronal damage in the C-fibers. The blue dashed line indicates the mean response for a healthy population.

\subsection{Optimal Spike Delays Parameters for A $\beta$ Fibers}

In this section, we target the second type of afferent fiber with our injury protocols. Due to myelination and larger diameter size, the conduction speed in the $A \beta$ fibers is much faster than in the $C$ fibers, and any stimulation of $A \beta$ fibers will reach the dorsal horn about $100 \mathrm{~ms}$ before the response from stimulation of the $C$ fibers. Since we measure pain with respect to the activity of the projection neurons during the $C$ response (recall Figure 3 ), the most likely way for injured activity on the $\mathrm{A} \beta$ fibers to affect the $\mathrm{C}$ response is to slow down the conduction speed or delay the spikes.

Figure 6A shows the change in the total area marker, $\mathrm{A}_{\text {total }}$, for delay times from $50 \mathrm{~ms}$ to $300 \mathrm{~ms}$. First, we point out that there exists an optimal delay time of $t^{\star}=125 \mathrm{~ms}$ that minimizes the total area, $A_{\text {total }}$. Figure $6 \mathrm{~B}$ shows the population responses for this optimal delay parameter as compared to a healthy response. Notice that stimulation on the $\mathrm{A} \beta$ fibers during the $C$ response ( $\sim 600-800 \mathrm{~ms}$ ) leads to an inhibition of the firing rate of the 
projection neurons due to the circuitry suggested by gate control theory. Namely, that $A \beta$ fibers synapse onto the inhibitory interneurons, which in turn synapse onto the projection neurons. In the original paper by Crodelle et al. [13], this is the mechanism by which the phenomenon known as pain inhibition works. The amount of pain inhibition depends on the timing of the $\mathrm{A} \beta$ stimulation, with optimal pain relief found for time delays that allow the $\mathrm{A} \beta$ pulse to occur near the beginning of the $\mathrm{C}$ response, as is the case for $t^{\star}=125 \mathrm{~ms}$.

A

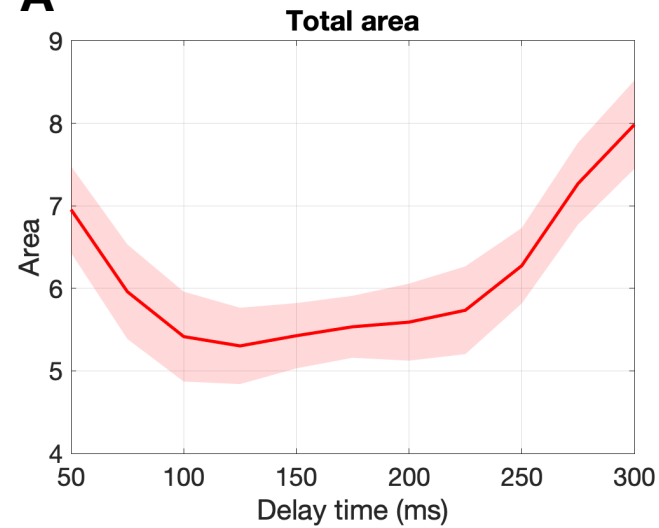

B

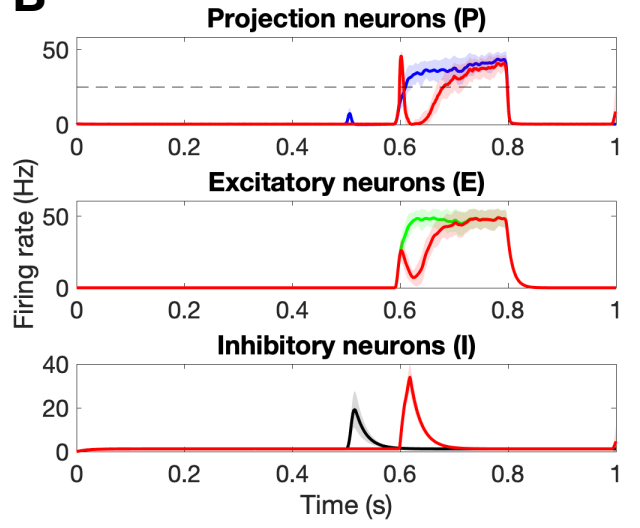

Figure 6. Damaging the $\mathrm{A} \beta$ fibers. (A): Damaging $50 \%$ of the $\mathrm{A} \beta$ fibers with different delay values to find the optimal delay time, $d^{\star}=125 \mathrm{~ms}$, that yields the minimal total area. Note that no $\mathrm{C}$ fibers were damaged and we are calculating the total area $A_{\text {total }}$. (B): Population responses of the projection, excitatory, and inhibitory neurons. The colored curves represent a healthy response, while the red curves represent the damaged response for the optimal delay time $d^{\star}=125 \mathrm{~ms}$. Note the shaded region is the standard deviation over 30 realizations.

Now that we established how pain might be affected by damaging the $\mathrm{A} \beta$ fibers, we go on to investigate possible interactions between damage on the $\mathrm{A} \beta$ fibers and on the C fibers.

\subsection{Damaging Both $A \beta$ and $C$ Fibers}

From Section 3.1, we saw that intermittent blocking and increased refractory in C-fibers both led to decreases in the response of the projection neurons, while evoked potentials led to an increased response. Since damaging the $\mathrm{A} \beta$ fibers (at particular delay times) also led to a decrease in the projection neuron response, it is redundant to consider the influence of two mechanisms to decrease pain. Instead, we consider evoked potentials on the $C$ fibers so as to study the interplay between an increased response due to the $C$ fibers and the pain inhibition evoked on the $A \beta$ fibers due to delayed spike damage.

Figure 7 shows the percent change of total area from the healthy case to the injured case for varying percentages of damaged $C$ and $A \beta$ fibers. We note that there is a sort of "tug-of-war" between the increased activity due to the evoked potentials on the $C$ fibers and the pain inhibition elicited by the delayed spikes on the A $\beta$ fibers. Interestingly, at the boundary of these two behaviors, illustrated by the white region in the left plot and the green curve in the contour plot, there is a balance between elicited spikes and spike inhibition such that there is no change in total area due to the different injuries on the two different fibers. This seems to suggest that small oscillations in the percentage of fibers injured near the boundary could potentially lead to oscillations in the observed pain response between increased pain and pain relief. 

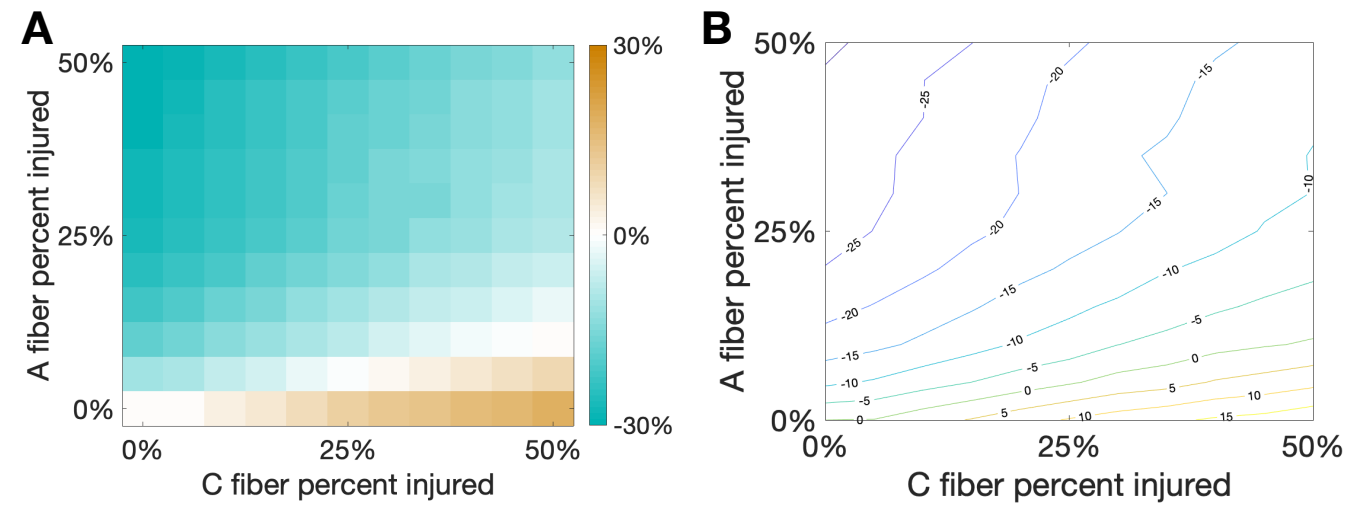

Figure 7. Damaging both $A \beta$ and $C$ fibers. In this simulation we consider a hybrid injury protocol in which signals from $A \beta$ fibers are delayed (with delay $d^{\star}=125 \mathrm{~ms}$ ) while evoked potentials are added to signals from $C$ fibers (with evoked probability of $10 \%$ ). This creates a "tug-of-war" between the two injury types that would respectively decrease/increase the pain marker $A_{\text {total }}$. The horizontal/vertical axes correspond to the percentage of damaged fibers from each type. (A): Heat map and (B): Contour curves are shown, with color indicating the fraction percent change of $A_{\text {total }}$ from the baseline pain response. Note that white regions in (A) and the contour curve associated to zero in (B) indicate injury combinations that would lead to the same $A_{\text {total }}$ observed in baseline, with one type of injury effectively cancelling out the other.

\section{Discussion and Conclusions}

\subsection{Overview of Results}

In this study, we use the computational model introduced by Crodelle et al. [12,13] to simulate pain processing in the dorsal horn under healthy and pathological conditions. Figure $1 \mathrm{~A}$ shows the underlying circuitry in which $\mathrm{C}$ fibers transmit pain signals from the peripheral to the spinal cord while $\mathrm{A} \beta$ fibers transmit touch stimuli. In normal conditions, projection neurons would integrate the input signals on the dorsal horn and transmit a stereotypical pain response to the cortex (Figure 1B). This outcome can change dramatically, however, if spike-train distortions associated with axonal damage [14-17] are introduced in the system (see Figure 2). In Figure 3, we define five quantitative painmarkers $\left[A_{\mathrm{total}}, A^{*}, \pi^{*}, \pi_{\max }, N_{C}\right]$ to characterize the observed changes in pain response caused by different injury protocols. Figures 4 and 5 show our results for when $C$-fibers are targeted with three different types of axonal injury (intermittent blocking, evoked potentials, and increased refractoriness). Figure 6 demonstrates another path for pain desensitization, where $\mathrm{A} \beta$ fibers are targeted and have their spike-trains delayed. Finally, in Figure 7 we consider a "tug-of-war" scenario where damaged $C$ fibers increase the painful signal (by evoked potentials) while damaged $A \beta$ fibers contribute in the opposite direction (by spike-train delays).

Our main goal throughout this work was to illustrate how a variety of non-trivial outcomes may emerge at the collective level from different types of injury protocols. Axonal damage in the C-fibers leading to evoked potentials/increased refractoriness translated to monotonic increase/decrease of all quantitative pain markers (Figures 4 and 5). However, the $N_{C}$ marker (that counts the number of times the pain signal crosses the typical $\pi_{\text {thresh }}=25 \mathrm{~Hz}$ threshold) behaves very differently for the intermittent blocking injury (see top-right plot in Figure 5). In this kind of injury, the spike trains are blocked intermittently, which leads to an oscillatory-kind of collective response (see Figure 4B). If the percentage of injured neurons is small(large), the oscillations remain above(below) the $\pi=25 \mathrm{~Hz}$ pain threshold, which in both cases lead to small $N_{C}$ counts. For an intermediate percentage, however, $N_{C}$ will achieve its maximum value. We posit that intermittent blocking and multiple crossings of pain threshold might be linked with throbbing/pulsating types of pain. Moreover, this feature does not require large percentages of injured neurons to be significant, which might explain its prevalence in clinical settings. 
Pain desensitization can also be achieved, in a more subtle way, by targeting A $\beta$ fibers. Specifically, we demonstrated that the occurrence of spike-train delays in A $\beta$ fibers will derange the expected response of the inhibitory neurons, which in turn, will lead to an overall weaker pain signal. Figure 6 shows that a delay of $d^{\star}=125 \mathrm{~ms}$ leads to a minimal area under the curve $\left(A_{\text {total }}\right)$ in the pain response. We conclude our exploration by creating a "tug-of-war" scenario in which the pain desensitization caused by these damaged Afibers competes with the sensitization caused by damaged C-fibers (that evoke potentials). As shown in Figure 7, these effects can cancel each other out (in the white boundary-like region between regimes). We conjecture that this delicate balance between the regimes could be offset, for instance, by fluctuations in the patient's circadian rhythm. In particular, healthy subjects typically experience the highest sensitivity to pain in the middle of the night and lowest in the afternoon, while patients suffering from neuropathy experience an approximately 12-h shift in their rhythmicity [12]. Altogether, improvements in the knowledge of the transmission of pain from the damaged nerve to the pain-processing center in the spinal cord may lead to better diagnostics and treatment protocols.

\subsection{Connection to Neuropathic Pain}

Our framework may provide insights into neuropathic pain, a form of pain-disorder that affects $7 \%-10 \%$ of the general population in which the underlying pathophysiology remains a contested topic [35]. Lesions on the peripheral fibers ( $A \beta, A \delta$ and $C$ fibers) are linked to distortions of sensory signals into the spinal cord and the brain $[35,36]$. One piece of the puzzle unraveled by micro-neurography studies is the presence of ectopic activity in primary afferent fibers [37,38]. If an afferent fiber is disconnected from the periphery due to an injury or a lesion (for example, neuroma $C$ fiber afferents), the remnants can generate ectopic activity [39] while the intact fibers may become hyperexcitable [40]. The enhanced excitability of spinal neurons produces may enable afferent fibers to activate second-order nociceptive neurons, generating the so-called central sensitization [41,42]. Thus, incorporating injured neuronal populations into the computational models of pain processing might shine new light on different types of stimulus-evoked pain (hyperalgesic vs. allodynic) or painful neuropathies associated with other diseases $[43,44]$.

\subsection{Limitations and Future Work}

The modeling effort presented here represents a high-level overview of potential mechanisms that might underlie the observed effects of axonal injury on pain experience. The study has several limitations. First, the circuitry in the spinal cord is much more complicated than presented here (see [45] for a review) and is still being unraveled $[4,46]$. In addition, the gate control theory of pain is flawed and doesn't account for many observed phenomena $[10,11]$, although many models use it as a basis for spinal circuitry $[5,6,8]$. The population-level firing-rate model used in this work does not reflect the reality that $\mathrm{DH}$ neurons are heterogeneous and exhibit distinctive action potential shapes and sizes, as well as different ion distributions. However, as shown in [13], the model uses distinct response curves for each population motivated by experiments and includes N-Methyl-D-Aspartate (NMDA)-like synapses by modeling the weight to the projection neurons as a function of the projection neuron activity. As a result, this simplified spinal-cord model can replicate known experimental pain phenomena such as pain inhibition and wind up.

Finally, our injury protocols should be regarded as a proof-of-concept at this stage since a single type of axonal injury (per fiber population) is unrealistic; Maia et al. [21], for instance, reported different types of focal axonal swelling distributions (which included normal transmission, filtering, reflection, and total blockage regimes). Other neuronal subpopulations (besides $\mathrm{C}$ fibers and $\mathrm{A} \beta$ fibers) may also be dysfunctional, which could lead to more sophisticated mixed effects and tugs-of-war. In future work, we expect to gather more experimental evidence/parameters to calibrate the model more realistically for different pain disorders. All things considered, the computational modeling of damaged 
neuronal assemblies is a promising avenue that may help unravel the myriad of responses observed in painful neuropathies.

Author Contributions: Conceptualization, J.C. and P.D.M.; methodology, J.C. and P.D.M.; software, J.C. and P.D.M.; validation, J.C. and P.D.M.; formal analysis, J.C. and P.D.M.; investigation, J.C. and P.D.M.; resources, J.C. and P.D.M.; data curation, J.C. and P.D.M.; writing-original draft preparation, J.C. and P.D.M.; writing-review and editing, J.C. and P.D.M.; visualization, J.C. and P.D.M.; project administration, J.C. and P.D.M.; funding acquisition, J.C. and P.D.M. All authors have read and agreed to the published version of the manuscript.

Funding: This research received no external funding.

Data Availability Statement: All significant MATLAB codes are made available at https: / / github. com/jcrodelle/damagedFibers (accessed on 15 April 2021).

Acknowledgments: J.C. and P.D.M. would like to thank the organizers of the Computational Biology seminar at the Courant Institute for facilitating this collaboration.

Conflicts of Interest: The authors declare no conflict of interest.

\section{Abbreviations}

The following abbreviations are used in this manuscript:

DH Dorsal Horn

FAS Focal Axonal Swelling

\section{Appendix A. More Details on the Spinal-Cord Model}

Here we present more details on the firing-rate model used in this work for pain processing in the spinal cord, a simplified version of the model developed in [13]. We assume that the average firing rate of the projection, inhibitory, and excitatory neuron populations, $f_{\mathrm{P}}, f_{\mathrm{I}}$, and $f_{\mathrm{E}}$, respectively, follow the dynamic equations

$$
\begin{aligned}
\frac{d f_{\mathrm{P}}}{d t} & =\frac{\mathrm{P}_{\infty}\left(g_{\mathrm{A} \beta \mathrm{P}} f_{\mathrm{A} \beta}(t)+g_{\mathrm{A} \delta \mathrm{P}} f_{\mathrm{A} \delta}(t)+\left(g_{\mathrm{CP}}+g_{\mathrm{NMDA}}\right) f_{\mathrm{C}}(t)+g_{\mathrm{EP}} f_{\mathrm{E}}-g_{\mathrm{IP}} f_{\mathrm{I}}\right)-f_{\mathrm{P}}}{\tau_{\mathrm{P}}}, \\
\frac{d f_{\mathrm{E}}}{d t} & =\frac{\mathrm{E}_{\infty}\left(g_{\mathrm{CE}} f_{\mathrm{C}}(t)-g_{\mathrm{IE}} f_{\mathrm{I}}\right)-f_{\mathrm{E}}}{\tau_{\mathrm{E}}}, \\
\frac{d f_{\mathrm{I}}}{d t} & =\frac{\mathrm{I}_{\infty}\left[g_{\mathrm{A} \beta \mathrm{I}} f_{\mathrm{A} \beta}(t)\right]-f_{\mathrm{I}}}{\tau_{\mathrm{I}}}
\end{aligned}
$$

where $t$ is time in seconds, and $\tau_{\mathrm{P}}=0.001 \mathrm{~s}, \tau_{\mathrm{E}}=0.01 \mathrm{~s}$, and $\tau_{\mathrm{I}}=0.02 \mathrm{~s}$ are the intrinsic time scales of each population. The populations communicate via average synapses that we refer to as weights $g_{i j}$ from presynaptic neuron populations $i(i=\mathrm{A} \beta, \mathrm{A} \delta, \mathrm{C}, \mathrm{P}, \mathrm{E}, \mathrm{I})$ to neuron population $j(j=\mathrm{P}, \mathrm{E}, \mathrm{I})$.

We also include N-methyl-D-aspartate (NMDA) type synapses from the C-fibers to the $\mathrm{P}$ population through modification of the synaptic weight, $g_{\mathrm{NMDA}}$ by the average firing rate of the $\mathrm{P}$ population

$$
\frac{d g_{\mathrm{NMDA}}}{d t}=\frac{\mathrm{M}_{\infty}\left(f_{\mathrm{P}}\right)-g_{\mathrm{NMDA}}}{\tau_{\mathrm{NMDA}}},
$$

where $\tau_{\mathrm{NMDA}}=1 \mathrm{~s}$.

The nonlinear response function for each population has the form

$$
y_{\infty}(x)=\max (y) \frac{1}{2}\left[1+\tanh \left(\frac{1}{\alpha_{y}}\left(x-\beta_{y}\right)\right)\right], y=P, E, I, M,
$$

mimicking the F-I curves found in [5]. Finally, for all pieces of the model, we use the same parameter values as in the original work [13]. 


\section{References}

1. Loeser, J.D.; Melzack, R. Pain: An overview. Lancet 1999, 353, 1607-1609. [CrossRef]

2. Garland, E.L. Pain processing in the human nervous system: A selective review of nociceptive and biobehavioral pathways. Prim. Care. 2012, 39, 561-571. [CrossRef] [PubMed]

3. Millan, M.J. Descending control of pain. Prog. Neurobiol. 2002, 66, 355-474. [CrossRef]

4. Todd, A.J. Neuronal circuitry for pain processing in the dorsal horn. Nat. Rev. Neurosci. 2010, 11, 823-836. [CrossRef]

5. Zhang, T.C.; Janik, J.J.; Grill, W.M. Modeling effects of spinal cord stimulation on wide-dynamic range dorsal horn neurons: Influence of stimulation frequency and GABAergic inhibition. J. Neurophysiol. 2014, 112, 552-567. [CrossRef] [PubMed]

6. Britton, N.F.; Skevington, S.M. A mathematical model of the gate control theory of pain. J. Theor. Biol. 1989, 137, 91-105. [CrossRef]

7. Aguiar, P.; Sousa, M.; Lima, D. NMDA Channels Together with L-Type Calcium Currents and Calcium-Activated Nonspecific Cationic Currents Are Sufficient to Generate Windup in WDR Neurons. J. Neurophys. 2010, 104, 1155-1166. [CrossRef]

8. Le Franc, Y.; Le Masson, G. Multiple firing patterns in deep dorsal horn neurons of the spinal cord: Computational analysis of mechanisms and functional implications. J. Neurophysiol. 2010, 104, 1978-1996. [CrossRef] [PubMed]

9. Melzack, R.; Wall, P.D. Pain mechanisms: A new theory. Science 1965, 150, 971-979. [CrossRef] [PubMed]

10. Mendell, L.M. Constructing and deconstructing the gate theory of pain. Pain 2014, 155, 210-216. [CrossRef] [PubMed]

11. Moayedi, M.; Davis, K.D. Theories of pain: From specificity to gate control. J. Neurophysiol. 2013, 109, 5-12. [CrossRef] [PubMed]

12. Crodelle, J.A.; Piltz, S.H.; Hagenauer, M.H.; Booth, V. Investigating circadian rhythmicity in pain sensitivity using a neural circuit model for spinal cord processing of pain. In Women in Mathematical Biology; Springer: Cham, Switzerland, 2016.

13. Crodelle, J.; Piltz, S.H.; Hagenauer, M.H.; Booth, V. Modeling the daily rhythm of human pain processing in the dorsal horn. PLoS Comput. Biol. 2019, 15, e1007106. [CrossRef]

14. Maia, P.D.; Raj, A.; Kutz, J.N. Slow-gamma frequencies are optimally guarded against neurodegenerative diseases and traumatic brain injury: Consequences for neural encoding and working memory. J. Comp. Neurosci. 2019, 47, 1-16. [CrossRef]

15. Maia, P.D.; Hemphill, M.A.; Zehnder, B.; Zhang, C.; Parker, K.K.; Kutz, J.N. Diagnostic tools for evaluating the impact of focal axonal swellings arising in neurodegenerative diseases and/or traumatic brain injury. J. Neurosci. Methods 2015, 253, $233-243$. [CrossRef]

16. Maia, P.D.; Kutz, J.N. Compromised axonal functionality after neurodegeneration, concussion and/or traumatic brain injury. J. Comp. Neurosci. 2014, 27, 317-332. [CrossRef]

17. Maia, P.D.; Kutz, J.N. Identifying critical regions for spike propagation in axon segments. J. Comp. Neurosci. 2014, 36, 141-155. [CrossRef]

18. Lusch, B.; Weholt, J.; Maia, P.D.; Kutz, J.N. Modeling cognitive deficits following neurodegenerative diseases and traumatic brain injuries with deep convolutional neural networks. Brain Cogn. 2018, 123, 154-164. [CrossRef]

19. Weber, M.; Maia, P.D.; Kutz, J.N. Estimating memory deterioration rates following neurodegeneration and traumatic brain injuries in a Hopfield Network Model. Front. Neurosci. 2017, 11, 623.

20. Morris, M.; Maia, P.D.; Kutz, J.N. Preventing neurodegenerative memory loss in Hopfield neuronal networks using cerebral organoids or external microelectronics. Comput. Math. Methods Med. 2017, 2017, 6102494.

21. Maia, P.D.; Kutz, J.N. Reaction time impairments in decision-making networks as a diagnostic marker for traumatic brain injury and neurodegenerative diseases. J. Comp. Neurosci. 2017, 42, 323-347. [CrossRef]

22. Kunert, J.; Maia, P.D.; Kutz, J.N. Functionality and robustness of injured connectomic dynamics in C. elegans: Linking behavioral deficits to neural circuit damage. PLoS Comp. Biol. 2017, 13, e1005261. [CrossRef] [PubMed]

23. Rudy, S.; Maia, P.D.; Kutz, J.N. Cognitive and behavioral deficits arising from neurodegeneration and traumatic brain injury: A model for the underlying role of focal axonal swellings in neuronal networks with plasticity. J. Syst. Int. Neurosci. 2016. [CrossRef]

24. Caro, X.J.; Galbraith, R.G.; Winter, E.F. Evidence of peripheral large nerve involvement in fibromyalgia: A retrospective review of EMG and nerve conduction findings in 55 FM subjects. Eur. J. Rheumatol. 2018, 5, 104-110. [CrossRef]

25. Zimmermann, M. Pathobiology of neuropathic pain. Eur. J. Pharmacol. 2001, 429, 23-37. [CrossRef]

26. Wilson, H.R.; Cowan, J.D. Excitatory and inhibitory interactions in localized populations of model neurons. Biophys. J. 1972, 12, 1-24. [CrossRef]

27. Ermentrout, G.B.; Terman, D.H. Mathematical Foundations of Neuroscience; Springer: Berlin/Heidelberg, Germany, 2010.

28. Reeve, A.J.; Walker, K.; Urban, L.; Fox, A. Excitatory effects of galanin in the spinal cord of intact, anaesthetized rats. Neurosci. Lett. 2000, 295, 25-28. [CrossRef]

29. Hulse, R.; Wynick, D.; Donaldson, L.F. Intact cutaneous C fibre afferent properties in mechanical and cold neuropathic allodynia. Eur. J. Pain 2010, 14, 565.e1-565.e10. [CrossRef]

30. Peyronnard, J.M.; Charron, L.F.; Lavoie, J.; Messier, J.P. Motor, sympathetic and sensory innervation of rat skeletal muscles. Brain Res. 1986, 373, 288-302. [CrossRef]

31. Le Bars, D.; Gozariu, M.; Cadden, S.W. Animal models of nociception. Pharmacol. Rev. 2001, 53, 597-652.

32. Smith, K.J. Conduction properties of central demyelinated and remyelinated axons, and their relation to symptom production in demyelinating disorders. Eye 1994, 8, 224-237. [CrossRef]

33. Gu, C. Rapid and reversible development of axonal varicosities: A new form of neural plasticity. Front. Mol. Neurosci. 2021, $14,1$. [CrossRef] [PubMed] 
34. Johnson, V.E.; Stewart, W.; Smith, D.H. Axonal pathology in traumatic brain injury. Exp. Neurol. 2013, 246, 35-43. [CrossRef] [PubMed]

35. Colloca, L.; Ludman, T.; Bouhassira, D.; Baron, R.; Dickenson, A.H.; Yarnitsky, D.; Freeman, R.; Truini, A.; Attal, N.; Finnerup, N.B.; et al. Neuropathic pain. Nat. Rev. Dis. Prim. 2017, 3, 17002 . [CrossRef] [PubMed]

36. Finnerup, N.B.; Haroutounian, S.; Kamerman, P.; Baron, R.; Bennett, D.L.; Bouhassira, D.; Cruccu, G.; Freeman, R.; Hansson, P.; Nurmikko, T.; et al. Neuropathic pain: An updated grading system for research and clinical practice. Pain 2016, 157, 1599-1606. [CrossRef]

37. Serra, J.; Bostock, H.; Solà, R.; Aleu, J.; García, E.; Cokic, B.; Navarro, X.; Quiles, C. Microneurographic identification of spontaneous activity in C-nociceptors in neuropathic pain states in humans and rats. Pain 2012, 153, 42-55. [CrossRef]

38. Kleggetveit, I.P.; Namer, B.; Schmidt, R.; Helås, T.; Rückel, M.; Ørstavik, K.; Schmelz, M.; Jørum, E. High spontaneous activity of C-nociceptors in painful polyneuropathy. Pain 2012, 153, 2040-2047. [CrossRef]

39. Tesfaye, S.; Boulton, A.J.; Dickenson, A.H. Mechanisms and management of diabetic painful distal symmetrical polyneuropathy. Diabetes Care 2013, 36, 2456-2465. [CrossRef]

40. Fields, H.L.; Rowbotham, M.; Baron, R. Postherpetic neuralgia: Irritable nociceptors and deafferentation. Neurobiol. Dis. 1998, 5, 209-227. [CrossRef] [PubMed]

41. Woolf, C.J. Central sensitization: Implications for the diagnosis and treatment of pain. Pain 2011, 152, S2-S15. [CrossRef]

42. Baron, R.; Hans, G.; Dickenson, A.H. Peripheral input and its importance for central sensitization. Ann. Neurol. 2013, 74, 630-636. [CrossRef]

43. Stavros, K.; Simpson, D.M. Understanding the etiology and management of HIV-associated peripheral neuropathy. Curr. HIV/AIDS Rep. 2014, 11, 195-201. [CrossRef] [PubMed]

44. Thakur, S.; Dworkin, R.H.; Haroun, O.M.; Lockwood, D.N.; Rice, A.S. Acute and chronic pain associated with leprosy. Pain 2015, 156, 998-1002. [CrossRef] [PubMed]

45. Duan, B.; Cheng, L.; Ma, Q. Spinal circuits transmitting mechanical pain and itch. Neurosci. Bull. 2018, 34, 186-193. [CrossRef] [PubMed]

46. Peirs, C.; Dallel, R.; Todd, A.J. Recent advances in our understanding of the organization of dorsal horn neuron populations and their contribution to cutaneous mechanical allodynia. J. Neural Transm. 2020, 127, 505-525. [CrossRef] 